(C) 2019 IEEE. Personal use of this material is permitted. Permission from IEEE must be obtained for all other uses, in any current or future media, including reprinting/republishing this material for advertising or promotional purposes, creating new collective works, for resale or redistribution to servers or lists, or reuse of any copyrighted component of this work in other works.

DOI: $10.23919 /$ PS.2019.8818018 


\title{
Spectrum/Space Switching and Multi-Terabit Transmission in Agile Optical Metro Networks
}

\author{
M. Svaluto Moreolo ${ }^{1}$, R. Martínez ${ }^{1}$, L. Nadal ${ }^{1}$, J. M. Fabrega ${ }^{1}$, N. Tessema ${ }^{2}$, N. Calabretta ${ }^{2}$, R. Stabile ${ }^{2}$ \\ P. Parolari ${ }^{3}$, A. Gatto ${ }^{3}$, P. Boffi ${ }^{3}$, G. Otero ${ }^{4}$, D. Larrabeiti ${ }^{4}$, J. A. Hernández ${ }^{4}$, P. Reviriego ${ }^{4}$, J. P. Fernández- \\ Palacios $^{5}$, V. López ${ }^{5}$, G. Delrosso ${ }^{6}$, C. Neumeyr ${ }^{7}$, K. Solis-Trapala ${ }^{8}$, G. Parladori ${ }^{9}$, G. Gasparini ${ }^{9}$ \\ ${ }^{1}$ Centre Tecnològic de Telecomunicacions de Catalunya (CTTC/CERCA), Castelldefels (Barcelona), Spain; ${ }^{2}$ Electrical Engineering \\ Department, Technische Universiteit Eindhoven (TU/e), Eindhoven, The Netherlands; ${ }^{3}$ Politecnico di Milano, Milan, Italy; \\ ${ }^{4}$ Universidad Carlos III de Madrid, Spain; ${ }^{5}$ Telefonica Global CTO, Madrid, Spain; ${ }^{6}$ VTT Technical Research Center of Finland; \\ ${ }^{7}$ Vertilas GmbH, Garching, Germany; ${ }^{8}$ EFFECT Photonics B.V., Eindhoven, The Netherlands; ${ }^{9}$ SM Optics, Milan, Italy \\ michela.svaluto@cttc.es
}

\begin{abstract}
An SDN-enabled modular photonic system architecture, including VCSEL-based bandwidth/bitrate variable transceivers, for multi-terabit capacity transmission and agile spectrum/space switching in optical metro networks is presented, providing the proposed technological solutions, programmability aspects and preliminary assessment.

Keywords: Switching Systems, Architectures and Network Integrations.
\end{abstract}

\section{INTRODUCTION}

Driven by the global IP traffic increase and the even more rapid growth of busy hour Internet traffic, future optical networks, and particularly the metropolitan area network (MAN) segment, should support ultra-high capacity and dynamicity, while lowering both capital and operational expenditures (CAPEX/OPEX). In fact, it is forecasted that Content Delivery Networks (CDNs) will carry $72 \%$ of Internet traffic by 2022 (56\% in 2017) and 33\% of service provider network capacity will be within the metro network by the same year (27\% in 2017) [1]. Thus, use cases to be considered and suitably addressed to meet the MAN evolutionary scenario requirements are i) cost-effective ultra-broadband transport and dynamic capacity adaptation, providing a pay-as-you-grow scheme, ii) efficient interconnection of distributed computation sites (e.g. CDN) within the MAN and iii) the ability of supporting massive events.

Programmability and modularity are key system features enabling the requested dynamicity and scalability. Both spectral and spatial resources should be exploited and efficiently managed to also comply with the stringent cost requirements imposed by MANs. Meanwhile, the proposed solutions should also target low power consumption and footprint. This is particularly relevant for the photonic technologies to be selected.

In the framework of EU-H2020 PASSION project, suitable programmable transceiver and switching/aggregation node architectures, as well as the photonic technologies to be exploited for their design, have been defined and are being implemented to deal with the cost/capacity requirements of the future MAN. In particular, a sliceable bandwidth and bitrate variable transceiver (S-BVT) modular architecture adopting dense photonic integration is proposed. Directly modulated (DM) vertical cavity surface emitting laser (VCSEL) sources with large bandwidth and operating wavelength within the C-band are combined with multi-channel coherent optical (CO) receivers to achieve multi-terabit capacity over metro network paths. A modular node architecture, equipped with photonic fundamental modules, is also proposed to enable handling express, added and dropped traffic in both space and spectrum dimensions. Additionally, programmability is considered for further integration in a software defined networking (SDN) control plane able to dynamically (re)-configure the S-BVT and node elements for flexible adaptation to the network conditions aiming at attaining an efficient resource usage. In this work, we present the SDN-enabled overall system architecture, providing details on the proposed technological solutions, programmability aspects and preliminary assessment.

\section{Programmable Modular System Architecture}

The programmable optical system proposed to target the requirements of future agile and high-capacity optical metro networks is depicted in Figure 1. The network nodes and the modular S-BVTs are configured by an SDN controller, by means of dedicated control agents. The accomplished modularity allows enhancing the supported capacity and providing advanced features according to the traffic/network needs. Thus, the S-BVT is suitably tailored to the metro network node type and can grow-as-needed to achieve the targeted capacity/flexibility, according to the evolution of the network. A very large 5G-supportive MAN based on real topologies is set as design target. It is made up of a layered composition of aggregation/distribution graphs with 5 hierarchical levels (HL), where HL5 corresponds to the bottom of the hierarchy: the edge nodes ( $>2400$ Base Stations and small Central Offices); the top metro-core level consists of a small mesh of HL2 and HL1 nodes performing aggregation, hosting services such as CDN and providing WAN connectivity. Lower level metro-aggregation nodes ( 400 HL4 nodes hosted in big Central Offices and transit hubs) have a simpler and more costeffective architecture than higher level nodes and are envisaged to be equipped with lower capacity S-BVTs. Metro-core nodes $(6 \mathrm{HL} 2 / 1$ nodes that route traffic from/to $>30 \mathrm{HL} 3$ transit nodes) are equipped with full-featured high-capacity S- 
BVTs with a modular design, enabling a finer aggregation/distribution of a larger amount of optical channels, the handling of large traffic flows and the support of more advanced functionalities, as will be detailed in Sec. II B (see also Fig. 3).

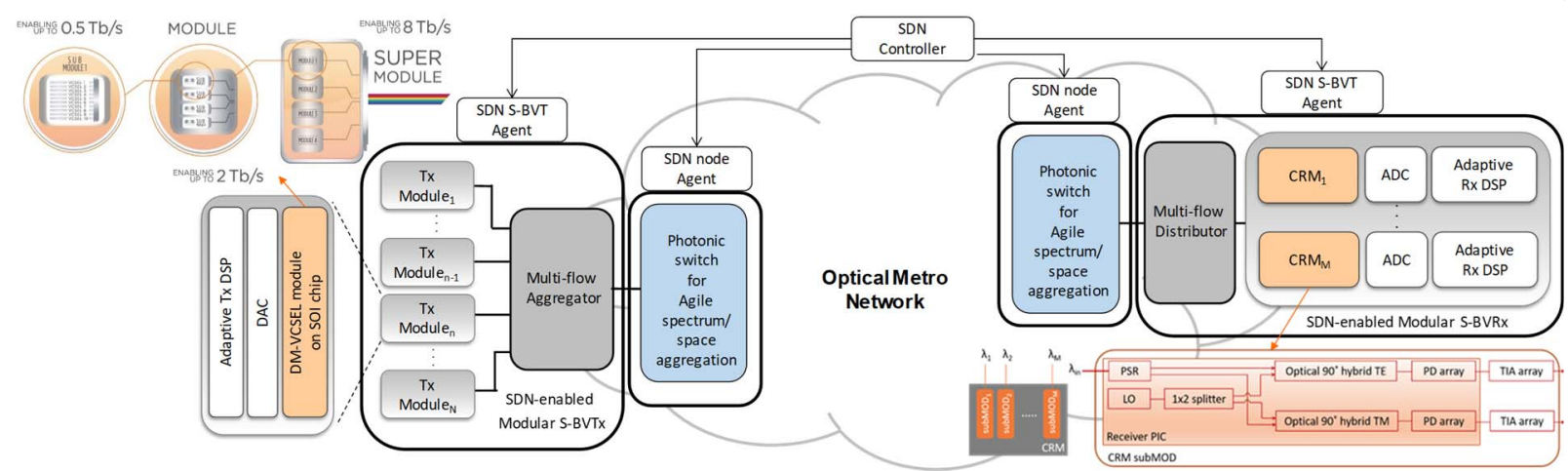

Fig. 1. Programmable (SDN-enabled) modular system architecture adopting photonic switching node (in blue) and S-BVT elements (in orange).

\section{A. $\quad$ S-BVT Architecture and Adopted Photonic Technologies}

The multiple modules at the S-BVT transmitter (S-BVTx) consist of DM short-cavity (SC) VCSELs characterized by

bandwidth larger than $18 \mathrm{GHz}$. The fundamental module is a silicon-on-insulator (SOI) chip integrating multiple

VCSELs (4 sub-modules of 10 VCSELs each) at operating wavelengths within the C-band (in the range $191.9 \mathrm{THz}-$ 195.9 THz, according to the ITU-T grid CH19-CH59), with $100 \mathrm{GHz}$ channel spacing, as shown in Fig. 2. In order to efficiently exploit the spectral resource, $25 \mathrm{GHz}$ channel spacing is considered integrating 4 modules. The generated flows are aggregated/disaggregated and switched in the spectrum and space dimensions at the node. The dropped traffic is finally recovered at the S-BVT receiver (S-BVRx) by means of multiple coherent receiver modules (CRM) integrated on PIC (right-hand inset of Fig.1). The adaptive digital signal processing (DSP) at the S-BVTx/Rx allows to suitably adapt the multiple data flows to the established connection requirements (e.g. bandwidth, traffic demand and quality of transmission). Adopting multicarrier modulation (MCM) - either double or single sideband (DSB, SSB) - with bit and power loading algorithms allows variable bandwidth/bitrate adaptation (per flow/slice) with sub-wavelength granularity [2]. This enables to support up to $50 \mathrm{~Gb} / \mathrm{s}$ per flow (single VCSEL), up to $8 \mathrm{~Tb} / \mathrm{s}$ (4 fundamental modules) and hundreds of $\mathrm{Tb} / \mathrm{s}$ (exploiting polarization/space dimensions) connections, thanks to the modular architecture (Fig. 2 bottom-left).
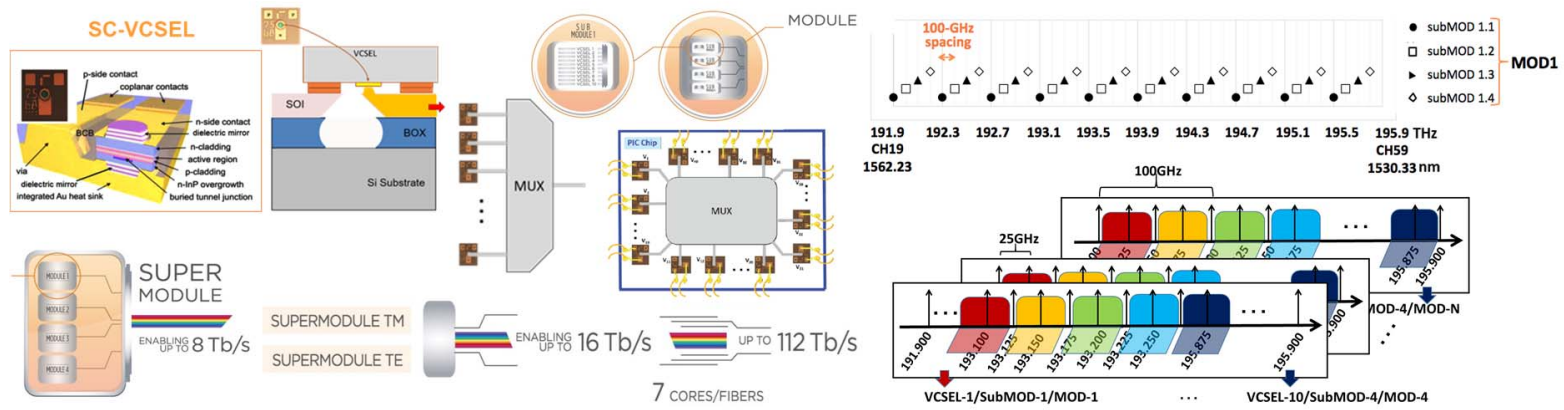

Fig. 2. S-BVT architecture with elements composing the (sub)-modules (left); VCSEL operating wavelength assignment per sub-module (up-right) and space/spectrum resource assignment per S-BVT element (bottom-right).

\section{B. Switching/Aggregation Node Architecture and Components Functionalities}

Referring to the hierarchical network architecture above described (see also Fig. 3(a)), at the HL4 nodes, routing and aggregation functionalities are realized with low cost switches adopting wavelength blockers, based on semiconductor optical amplifier (SOA), for a simple add/drop functionality, as shown in Fig. 3(b). The node typically consists of a demultiplexing arrayed waveguide grating (AWG) at the input; 1:2 splitters are used to tap the drop signal, which is broadcasted to a number of splitters to lower level (HL5) nodes. Figure 3(c) shows the switching functionalities carried out by the components at the HL3 nodes. HL2/HL1 nodes have similar architecture as they require the same functionalities as HL3 nodes. The photonic switching module (PSM) provides space-based switching of the traffic at any input port to any output port, which are either assigned to express out path or dropped traffic path. The aggregate/disaggregate switches do the routing of the dropped traffic either to the multicast switch (MCS) or add switch, thanks to the 1x2 WSS per input port. The add switch provides merging and aggregating inputs originating from S-BVTs or directly from the aggregate/disaggregate switch to the add ports of the PSM. The drop part diverts some of the link traffic to drop it at the S-BVRx side. For this purpose, the MCSes enable colorless and contention-less switching to efficiently use the available CRMs. For the uplink traffic, a WSS-based add switch is used to enable a contention-less, directionless and colorless addition of the traffic to the HL3 nodes. A wavelength multiplexer might be used before the add switch for aggregation purposes. This node architecture enables efficiently utilize the resources bundling traffic in space and spectrum; capacity upgrade is achieved repeating the same blocks, thanks to the flexible and modular design. The ultra-high capacity interconnection is carried out via multi-core fibers, facilitated by the fan-out and fan-in structures. 
(a)

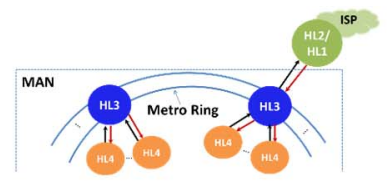

(b)

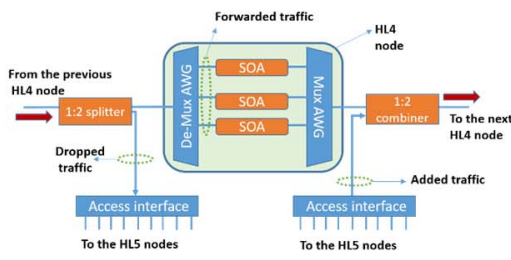

(c)

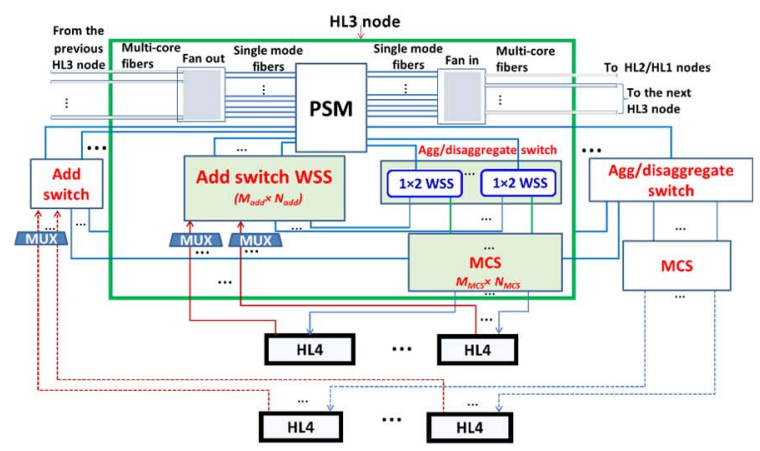

Fig. 3. Node architectures considering different MAN hierarchical levels (a): HL4 SOA-based node (b) and HL3 (or HL2/1) modular node (c).

\section{Preliminary Assessment and Programmability Aspects}

Taking into account the target hierarchical MAN, the most challenging scenario to be addressed in terms of required capacity is the cost-effective ultra-broadband transport providing a feasible pay-as-you-grow scheme to deal with future traffic demands. The proposed system architecture and technologies have been assessed accordingly, considering a target capacity per flow (i.e. per VCSEL) of $50 \mathrm{~Gb} / \mathrm{s}$ (to achieve the aggregated capacities as in Fig. 1 and 2) over a path traversing multiple nodes with distance between two consecutive nodes of either $35 \mathrm{~km}$ or $65 \mathrm{~km}$. HL4 node filtering is based on $100 \mathrm{GHz}$ AWG (2 per each node), while HL3 and HL2/1 nodes include both AWG and 25-GHz WSS.

SC-VCSEL technology combined with CO-Rx allows achieving very promising results thanks to the adoption of direct SSB multicarrier modulation with adaptive BL [2]. $50 \mathrm{~Gb} / \mathrm{s}$ per flow are supported over an amplified (EDFA with $6 \mathrm{~dB}$ noise figure) multi-hop path of $260 \mathrm{~km}$ (SSMF span of $65 \mathrm{~km}$ ) traversing up to $5 \mathrm{WSS}$, in linear propagation regime for optical signal-to-noise ratio (OSNR) values above $30 \mathrm{~dB}$. Larger paths (up to $735 \mathrm{~km}$ ) can be reached if the connection is characterized by shorter hop distances $(35 \mathrm{~km})$, assuming the same number of filtering stages. These results have been achieved, considering a measured chirp factor $\alpha$ of 3.7 [3]. Then, we have assessed the cascading effect of a single channel DMT signal passing through multiple AWG and WSS filters over an established MAN connection, taking into account node and transceiver architectures. Considering 2 WSSes (e.g. for SSB filtering at the S-BVTx and at the Rx side), over $50 \mathrm{~Gb} / \mathrm{s}$ connections including up to 14 AWG filters (e.g 2 at the S-BVT and 6 hops HL4-HL3 path, found to be worstcase mean value) can be established with $30 \mathrm{~dB}$ OSNR; while the required OSNR value should be at least $35 \mathrm{~dB}$ to support a $50 \mathrm{~Gb} / \mathrm{s}$ connection including 4 WSSes (at S-BVT and HL2/1 filtering) and 6 AWGs (at S-BVT and at the node).

To fully integrate the programmability and softwarization in the optical system to exploit their intrinsic advantages, it is crucial considering the peculiarities of the elements and devices to be configured, as well as the limitations/potentialities of the adopted photonic technologies. This also implies carefully identifying the parameters that can be accessible/programmable to define the information model to be used by the SDN controller. This model identifies, in an abstracted way, the relevant information (e.g. (sub)-module ID, wavelength, bandwidth, DSP parameters, etc.) to achieve the actual configuration of the underlying devices and network elements, for a suitable adaptation to multiple rate/reach, according to traffic demand and selected path, spatial/spectral aggregation, resource/dimension usage, slice-ability and other advanced functionalities [4]. This fosters the definition of the control interactions (i.e., interfaces and protocols) between the centralized SDN controller and the elements (i.e., agents), which directly handle the device configuration.

\section{CONCLUSIONS}

A modular programmable system including space/spectrum switching node and S-BVT architectures adopting suitable photonic technologies (i.e. long-wavelength SC-VCSELs and dense photonic integration) is presented to ease scalability, flexibility and on-demand adaptation for an efficient network resource usage addressing the challenges of future MANs.

\section{ACKNOWLEDGMENT}

Work funded by the EU H2020 PASSION project GA 780326 and the Spanish AURORAS (RTI2018-099178-B-I00).

\section{REFERENCES}

[1] Cisco Visual Networking Index: Forecast and Trends, 2017-2022, Nov 2018.

[2] M. Svaluto Moreolo et al., "VCSEL-based sliceable bandwidth/bitrate variable transceivers," Proc. SPIE-PWO, San Francisco, CA (USA), Feb. 2019.

[3] M. Rapisarda, et al., "Impact of Chirp in High-Capacity Optical Metro Networks Employing Directly-Modulated VCSELs," Photonics, vol. 5 (4), 51, 2018.

[4] R. Martinez et al., "Proof-of-Concept validation of SDN-controlled VCSEL-based S-BVTs in flexi-grid optical metro networks," Proc. OFC 2019, San Diego, CA (USA), March 2019. 